\title{
On Resonant Non Linearly Coupled Oscillators with Two Equal Frequencies
}

\author{
Martin Kummer \\ Department of Mathematics, The University of Toledo, Toledo, Ohio 43606, USA
}

\begin{abstract}
This paper contains a detailed study of the flow that the classical Hamiltonian

$$
H=\frac{1}{2}\left(x_{1}^{2}+y_{1}^{2}\right)+\frac{1}{2}\left(x_{2}^{2}+y_{2}^{2}\right)+\mathcal{O}_{3}
$$

induces in $R^{4}, \mathcal{O}_{3}$ representing a convergent power series that begins with a third order term.

In particular the existence and stability of periodic orbits is investigated.
\end{abstract}

\section{Introduction}

This paper contains a detailed description of the flow that the classical Hamiltonian (1.1) induces in its phase space $R^{4}$. The Hamiltonian describes two harmonic oscillators with equal frequencies that are coupled through a nonlinear force. This force can be quite general. The only requirement is that it derives from a potential that is represented by a convergent power series in the position and momentumvariables of the oscillators.

Our investigation was stimulated by the special case of the Hénon-Heiles Hamiltonian. A detailed study of that special case can be found in Ref. [1]. Ref. [1] also contains a general result about Hamiltonians of the form (1.1), namely: conditions are formulated under which Moser's twist theorem implies the existence of infinitely many invariant tori on each energy surface (compare the theorem on p. 313). As a side result of our investigation it is shown (Section 5) that these conditions cannot be quite correct and a correction is suggested.

Our detailed investigation of the flow that the Hamiltonian (1.1) induces in $R^{4}$ also uses as its main tool the Gustavson normal form. Because the symplectic transformations that leave the leading term of the Hamiltonian (1.1) invariant constitute exactly the group $U(2)$, the Gustavson normal form is best viewed as a function over the Lie algebra of that group. We split the Hamiltonian into two parts: the unperturbed or truncated Hamiltonian consisting of the sum of the leading term and of the first nonvanishing term of the Gustavson normal form, 
which we assume to be a polynomial in the generators of the Lie algebra $u(2)$ of degree $n$, and a perturbation which constitutes the (suitably transformed) remaining part of the power series in (1.1). In Sections 1-3 we keep $n$ quite general, postponing the most important case $n=2$ to Section 4 . In Section 1 we investigate the question of the uniqueness of the unperturbed Hamiltonian and find that exactly the subgroup $S U(2)$ of $U(2)$ connects different equivalent unperturbed Hamiltonians with each other. Here two unperturbed Hamiltonians are called equivalent if they are obtained by a canonical transformation from the same Hamiltonian (1.1). Moreover, the representation that this group induces in the space of polynomials of degree $n$ over $U(2)$ is nothing but the representation

$(0)+(1)+\ldots+(n)$

of $S O(3)$.

Another ambiguity in the coefficients of the unperturbed Hamiltonian stems from the fact that in the problem at hand, the sum of the squares of the generators of $S U(2)$ is the square of the fourth generator of $U(2)$, which therefore has great resemblance with the angular momentum of a top. In the case $n=2$ this relationship between our problem and the problem of the asymmetric top is described in exact terms in Theorem 3.

The flow of the unperturbed Hamiltonian in $R^{4}$ is completely determined by the flow that it induces on the unit sphere $S^{2}$ in the three dimensional space $s u(2) \approx s o(3)$, which we identify with $R^{3}$. Of particular interest are the critical points (abbreviated: c.p.'s) of this flow, which in turn agree with the c.p.'s of the unperturbed Hamiltonian, viewed as a function over $S^{2}$. General formulae for the indices of those c.p.'s are developed.

For the case $n=2$, a complete account of the relationship between the unperturbed Hamiltonian and the possible stable and unstable c.p.'s that it possesses on $S^{2}$ is presented. Needless to say, this task is greatly simplified by choosing from among all possible unperturbed equivalent Hamiltonians the simplest one (the so-called "diagonalized Gustavson normal form").

The theory will be illustrated here with the help of the familiar example of the Hénon-Heiles model, and the results will be compared with those of Ref. [1]. It turns out that we obtain one additional c.p., which, to the author's knowledge, has not yet been discovered due to a special choice of coordinates. These coordinates are like those obtained by the stereographic projection of $S^{2}$ regular everywhere except in exactly one point. (See Fig. 1.)

In Section 3 we will show that to each c.p. that the unperturbed Hamiltonian possesses on $S^{2}$, there corresponds a one-parametric family of periodic solutions of the equations associated with the full Hamiltonian. The stability of these periodic solutions will be investigated with the help of Moser's twist theorem (Theorem 1). A stable c.p. represents a family of stable periodic solutions only if the expression (3.5) is nonzero. It turns out, however, that for a certain type of c.p.'s that we call "c.p.'s of the second kind" and whose existence is partly due to the vanishing of certain coefficients in the unperturbed Hamiltonian (for a more exact definition see Section 4), their stability is already sufficient for the stability of the corresponding periodic solutions (Theorem 4). 


\section{General Considerations}

As announced in the introduction, we intend to study the flow induced in $R^{4}$ by Hamiltonians of the form

$$
H=\frac{1}{2}\left(x_{1}^{2}+y_{1}^{2}\right)+\frac{1}{2}\left(x_{2}^{2}+y_{2}^{2}\right)+\sum_{k=3}^{\infty} H_{k}\left(x_{1}, y_{1}, x_{2}, y_{2}\right),
$$

where $H_{k}$ is a homogeneous polynomial of degree $k$ in the variables $x_{1}, y_{1}, x_{2}, y_{2}$ and the infinite sum (1) represents some function that is real analytic at the origin of $R^{4}$.

The fundamental symplectic 2 -form corresponding to (1) is

$$
\omega=\sum_{k=1}^{2} d y_{k} \wedge d x_{k} \text {. }
$$

It turns out that the variables $z_{k}, \bar{z}_{k}$, and $N_{k}, \alpha_{k}(k=1,2)$ defined by

$$
\left.\begin{array}{l}
z_{k}=(1 / \sqrt{2})\left(x_{k}+i y_{k}\right)=N_{k}^{1 / 2} i e^{-i \alpha_{k}} \\
\bar{z}_{k}=\text { conjugate complex of } z_{k}, N_{k}=\left|z_{k}\right|^{2} \quad(k=1,2)
\end{array}\right\}
$$

are particularly well adapted to the problem at hand.

In these variables the fundamental 2 -form $\omega$ becomes

$$
\omega=(1 / i) \sum_{k=1}^{2}\left(d z_{k} \wedge d \bar{z}_{k}\right)=\sum_{k=1}^{2} d N_{k} \wedge d \alpha_{k} .
$$

For any two (complex valued) analytic functions $f, g$, defined on the phase space, we define the bracket

$$
[f, g]=\sum_{k=1}^{2}\left(\left(\partial f / \partial z_{k}\right)\left(\partial g / \partial \bar{z}_{k}\right)-\left(\partial g / \partial z_{k}\right)\left(\partial f / \partial \bar{z}_{k}\right)\right)
$$

such that the time rate of change of a function $f$ under the flow induced by $H$ in $R^{4}$ is given by

$$
\dot{f}=i[H, f] \text {. }
$$

Here the dot denotes derivative with respect to the time $t$.

The study of the flow in $R^{4}$ corresponding to the Hamiltonian (1) is facilitated if we bring (1) into Gustavson normal form. For this purpose it proves to be advantageous to introduce the following quadratic expressions in the $z$-variables:

$$
M_{k}=\frac{1}{2} z^{\dagger} \sigma_{k} z, \quad(k=0,1,2,3),
$$

where

$$
\sigma_{0}=\left(\begin{array}{ll}
1 & 0 \\
0 & 1
\end{array}\right), \quad \sigma_{1}=\left(\begin{array}{rr}
0 & -i \\
i & 0
\end{array}\right), \quad \sigma_{2}=\left(\begin{array}{ll}
0 & 1 \\
1 & 0
\end{array}\right), \quad \sigma_{3}=\left(\begin{array}{rr}
-1 & 0 \\
0 & +1
\end{array}\right)
$$

are the Pauli matrices and $z, z^{\dagger}$ denote the column matrix $\left(\begin{array}{l}z_{1} \\ z_{2}\end{array}\right)$ and its adjoint $\left(\bar{z}_{1}, \bar{z}_{2}\right)$, respectively.

Because $M_{0}$ will play a special role analogous to the magnitude of the angular momentum of a top we will also denote it by $J$. 
The following relations are immediate consequences of the definitions

$$
\begin{aligned}
& M_{1}=\operatorname{Im}\left(\bar{z}_{1} z_{2}\right), \quad M_{2}=\operatorname{Re}\left(\bar{z}_{1} z_{2}\right), \quad M_{3}=\frac{1}{2}\left(N_{2}-N_{1}\right) \\
& M_{0} \equiv J=\frac{1}{2}\left(N_{1}+N_{2}\right), \\
& M_{1}^{2}+M_{2}^{2}+M_{3}^{2}=J^{2} .
\end{aligned}
$$

The $M_{k}$ 's $(k=0,1,2,3)$ obey bracket relations that are isomorphic to those of $u(2)$. In particular, we have:

$$
\left[M_{1}, M_{2}\right]=i M_{3}
$$

and cyclic, i.e. the $M_{k}$ 's $(k=1,2,3)$ span the subalgebra $s u(2) \approx s o(3)$.

Now the normal form theorem [Ref. 2,3] states that there exists a formal transformation of variables

$$
z_{k} \rightarrow \hat{z}_{k} \quad(k=1,2),
$$

such that in the new variables $\hat{z}_{k}(k=1,2)$ the Hamiltonian $H$ has the form $2 J+K$, where $K$ is a formal power series in the variables $\hat{z}_{k}(k=1,2)$ which has zero bracket with the leading term $J$.

In terms of our generators of $u(2)$, however, this means that $K$ has the form

$$
K=\sum_{m=2}^{\infty} K_{m}\left(J, M_{1}, M_{2}, M_{3}\right)
$$

where $K_{m}$ is a homogeneous polynomial of degree $m$, defined on the Lie algebra $u(2)$, and the infinite sum is purely formal. Actually, the $M_{k}$ 's $(k=0,1,2,3)$ should carry carets because they are defined as in (7) with $z$ replaced by $\hat{z}$. However, after the transformation into (11) has been accomplished, we may drop the carets again.

In order to study the analytic Hamiltonian (1), we cannot use the purely formal expression (11). We must truncate the transformation of variables at some finite power in order to make sure that the transformation itself, as well as the transformed Hamiltonian, are still analytic in a suitable neighborhood of the origin of $R^{4}$.

In the following we will assume that the transformation of variables leading to the Gustavson normal form has been truncated in such a way that (1) in the new variables assumes the form

$$
H=2 J+K_{n}\left(J, M_{1}, M_{2}, M_{3}\right)+\mathcal{O}_{2 n+1},
$$

where $K_{n}$ is the first term in the normal form (11) that does not vanish identically and $\mathcal{O}_{2 n+1}$ stands for a convergent power series in the new variables that begins with a homogeneous polynomial of degree $2 n+1$.

Of course, in most cases we will have $n=2$, but while discussing general properties of the flow corresponding to the Hamiltonian (1), we will keep $n$ quite general and postpone the case $n=2$ to Section 4 .

Our strategy is to drop the term $\mathcal{O}_{2 n+1}$ first and study the flow of the truncated or unperturbed Hamiltonian and only afterwards to find conditions under which at least some features of the flow obtained in this way carry over to the flow induced in $R^{4}$ by the original Hamiltonian. 
At this stage, let us note that the coefficients of the polynomial $K_{n}$ in (12) are by no means uniquely determined. There are two reasons why this is so. First, if $n$ is even, then

$$
\left(J^{2}-M_{1}^{2}-M_{2}^{2}-M_{3}^{2}\right)^{n / 2}
$$

is a polynomial of degree $n$ in the $M_{k}$ 's $(k=0,1,2,3)$ that vanishes identically. Hence, if $a_{k_{0} k_{1} k_{2} k_{3}}$ is the coefficient of $M_{0}^{k_{0}} M_{1}^{k_{1}} M_{2}^{k_{2}} M_{3}^{k_{3}}$ in $K_{n}$ and $c_{k_{0} k_{1} k_{2} k_{3}}$ the corresponding coefficient in the polynomial (13), then $H_{n}$ is unaltered by a translation

$$
a_{k_{0} k_{1} k_{2} k_{3}} \rightarrow a_{k_{0} k_{1} k_{2} k_{3}}+\mu C_{k_{0} k_{1} k_{2} k_{3}}, \quad \mu \in R
$$

of its coefficients.

Secondly - and this will be of great importance in the following - we remind the reader that any transformation of the $z$-variables

$$
\hat{z}=U z,
$$

where $U$ belongs to the group $S U(2)$, is symplectic [i.e. leaves (4) invariant] and simultaneously induces a rotation $R(U)$ of the vector $\boldsymbol{M}=\left(M_{1}, M_{2}, M_{3}\right)$ :

$$
\hat{\boldsymbol{M}}=R(U) \boldsymbol{M}
$$

by means of the formula

$$
U^{\dagger} \sigma_{k} U=\sum_{l=1}^{3} R(U)_{k l} \sigma_{l} .
$$

Obviously, the linear space of homogeneous polynomials defined on the Lie algebra $u(2)$ is carried into itself by such transformations.

\section{The Flow of the Truncated (Unperturbed) Hamiltonian}

In this section we study some features of the flow corresponding to the Hamiltonian

$$
H_{0}=2 J+K_{n}\left(J, M_{1}, M_{2}, M_{3}\right),
$$

where $K_{n}$ is of degree $n$ in the variables $M_{k}(k=0,1,2,3)$. Introducing the unit vector $\boldsymbol{x}$ in the direction of $\boldsymbol{M}$ :

$$
\boldsymbol{M}=J \boldsymbol{x}, \quad \boldsymbol{x}=(x, y, z),
$$

we have

$$
K_{n}(J, M)=J^{n} K^{(n)}(\boldsymbol{x}),
$$

where $K^{(n)}(\boldsymbol{x})=K_{n}(1, \boldsymbol{x}) . K^{(n)}(\boldsymbol{x})$ is a polynomial of degree $n$, defined on the unit sphere $S^{2}$, and therefore it has a representation

$$
K^{(n)}(\boldsymbol{x})=\sum_{l=0}^{n} \sum_{m=-l}^{l} a_{l m} Y_{l m}(\boldsymbol{x}),
$$

where $Y_{l m}(x)$ are the usual spherical harmonics. Because $K^{(n)}(x)$ and $J$ are integrals of the Hamiltonian (1), it is clear that this Hamiltonian induces a flow on $S^{(2)}$, whose orbits are the level curves of $K^{(n)}(\boldsymbol{x})$. We will show in a moment that this flow on $S^{2}$ determines the flow in the original phase space $R^{4}$ completely. Antici- 
pating this result for a moment, we note that the critical points (c.p.'s) of the flow induced by (1) on $S^{2}$ are the c.p.'s of the function $K^{(n)}$ on $S^{2}$. Accordingly the end point of unit vector $\boldsymbol{e}$ is a critical point if there exists a real number $\lambda$ such that

$$
\left.\nabla_{x} K^{(n)}\right|_{x=e}=\lambda e .
$$

This is also seen by observing that as a consequence of $(1.10)$ the flow on $S^{2}$ is governed by the differential equations

$$
d \boldsymbol{x} / d \tau=\nabla_{\boldsymbol{x}} K^{(n)} \times \boldsymbol{x}
$$

where $\tau=J^{n-1} t$ and $\times$ denotes the usual cross product.

In order to study the nature of a c.p., we will make use of the following quadratic form over $R^{3}$ :

$$
Q(x)=(x,(\mathfrak{A}(e)-\lambda) x) .
$$

Here (, ) denotes the usual inner product of $R^{3}$ and $\mathfrak{U}(\boldsymbol{e})$ is the linear transformation of $R^{3}$ associated with the Hessian of $K^{(n)}$ evaluated at $e$. A c.p. is called degenerate if $Q(x)$, restricted to the plane of vectors orthogonal to $e$, is semi-definite. Obviously, a nondegenerate c.p. is stable or unstable (elliptic or hyperbolic) depending on whether $Q(x)$, restricted to that plane, is definite or indefinite. In the following we mean by a c.p. a nondegenerate c.p., unless stated otherwise.

If $\boldsymbol{e}$ is the position vector of a c.p. on $S^{2}$, then, by a special unitary transformation of the $z$-variables (compare 1.15-17), it can always be rotated into the negative 3-direction. Assuming that such a transformation has been performed, $-\boldsymbol{e}_{3}=(0,0,-1)$ is a c.p., and consequently we have

$$
\left(\partial K^{(n)} / \partial x\right)_{\boldsymbol{x}=-\boldsymbol{e}_{3}}=\left(\partial K^{(n)} / \partial y\right)_{\boldsymbol{x}=-\boldsymbol{e}_{3}}=0 .
$$

It is easy to see that our criterion for determining the nature of the c.p. reduces to the determination of the sign of the expression

$$
\Delta(\lambda)=\left(\lambda-A_{11}\right)\left(\lambda-A_{22}\right)-A_{12}^{2},
$$

where

$$
A_{11}=\left(\partial^{2} K^{(n)} / \partial x^{2}\right)_{\boldsymbol{x}=-\boldsymbol{e}_{3}}, \quad A_{22}=\left(\partial^{2} K^{(n)} / \partial y^{2}\right)_{\boldsymbol{x}=-\boldsymbol{e}_{3}}, \quad A_{12}=\left(\partial^{2} K^{(n)} / \partial x \partial y\right)_{\boldsymbol{x}=-\boldsymbol{e}_{3}} .
$$

In short, the index for our c.p. is given by the formula

$$
\text { ind }\left(-e_{3}\right)=\operatorname{sgn} \Delta(\lambda) \text {. }
$$

We also observe that the special unitary transformation of the $z$-variables that brings $\boldsymbol{e}$ into the negative 3 -direction can always be chosen in such a way that $A_{12}=0$ in (9).

We now proceed to relate the flow induced by (1) on the unit sphere $S^{2}$ to the flow in the original phase space $R^{4}$. To this end we start from the canonical variables $\left(N_{k}, \alpha_{k}\right)(k=1,2)$ as introduced in (1.3) and immediately switch to the new variables

$$
J, \quad \psi=\alpha_{1}+\alpha_{2} ; \quad M_{3}, \quad \phi=\alpha_{2}-\alpha_{1},
$$


in which the fundamental 2-form has the following representation:

$$
\omega=d J \wedge d \psi+d M_{3} \wedge d \phi .
$$

This coordinate system is undefined for $M_{3}= \pm J$, i.e., for two 2-planes in $R^{4}$ which correspond to the north and south pole of $S^{2}$. Using (1.3) and (1.8), we find

$$
M_{1}=\sqrt{J^{2}-M_{3}^{2}} \sin \phi, \quad M_{2}=\sqrt{J^{2}-M_{3}^{2}} \cos \phi
$$

or

$$
x=\sqrt{1-z^{2}} \sin \phi, \quad y=\sqrt{1-z^{2}} \cos \phi .
$$

Hence, introducing $\theta$ by $\cos \theta=z$, we see that $\phi, \theta$ are polar coordinates on $S^{2}$ and that $\phi, \theta, \psi, 2 J^{1 / 2}$ are exactly the kind of polar coordinates in $R^{4}$ that result if $S^{3}$ is viewed as covering space of the rotation group $S O(3)$; i.e., $\phi, \theta, \psi$ are the Euler angles whose values are restricted to the following intervals:

$$
\begin{aligned}
& 0 \leqq \phi<2 \pi \\
& 0 \leqq \theta<\pi \\
& 0 \leqq \psi<4 \pi .
\end{aligned}
$$

Indeed, by using (1.3), (1.8), (2), and (12) we find that the matrix

$$
Z=\frac{1}{2} J^{-1 / 2}\left(\begin{array}{cc}
y_{2}+i x_{2} & x_{1}+i y_{1} \\
-x_{1}+i y_{1} & y_{2}-i x_{2}
\end{array}\right)=(2 J)^{-1 / 2}\left(\begin{array}{cc}
i \bar{z}_{2} & z_{1} \\
-\bar{z}_{1} & -i z_{2}
\end{array}\right)
$$

in terms of the angles $\phi, \theta, \psi$ is identical with the very member of $S U(2)$ that represents a rotation with Euler angles $\phi, \theta, \psi$, namely

$$
Z=\left(\begin{array}{ll}
e^{i(\phi+\psi) / 2} \cos \frac{1}{2} \theta & i e^{i(\phi-\psi) / 2} \sin \frac{1}{2} \theta \\
i e^{i(\psi-\phi) / 2} \sin \frac{1}{2} \theta & e^{-i(\phi+\psi) / 2} \cos \frac{1}{2} \theta
\end{array}\right) .
$$

This identity exhibits the relationship between the old coordinates of $R^{4}$ and the new coordinates $J, \phi, \theta, \psi$. More explicitly, we have

$$
\left.\begin{array}{l}
x_{1}=2 J^{1 / 2} \sin ((\psi-\phi) / 2) \sin \frac{1}{2} \theta \\
y_{1}=2 J^{1 / 2} \cos ((\psi-\phi) / 2) \sin \frac{1}{2} \theta \\
x_{2}=2 J^{1 / 2} \sin ((\psi+\phi) / 2) \cos \frac{1}{2} \theta \\
y_{2}=2 J^{1 / 2} \cos ((\psi+\phi) / 2) \cos \frac{1}{2} \theta
\end{array}\right\}
$$

Although the variables $J, \phi, \theta, \psi$ are not quite canonical, because the fundamental 2-form expresses itself in these coordinates as follows:

$$
\omega=d J \wedge d \psi+(\cos \theta d J-J \sin \theta d \theta) \wedge d \phi,
$$

it is easy to write down the corresponding Hamiltonian equations. We find

$$
\begin{aligned}
& \dot{\psi}=2+J^{n-1}\left[n K^{(n)}(\boldsymbol{x})+\cot \theta \partial K^{(n)} / \partial \theta(\boldsymbol{x})\right] \\
& \dot{\phi}=-\left(J^{n-1} / \sin \theta\right) \partial K^{(n)} / \partial \theta(\boldsymbol{x}) \\
& \dot{\theta}=\left(J^{n-1} / \sin \theta\right) \partial K^{(n)} / \partial \phi(x),
\end{aligned}
$$

where $\boldsymbol{x}=(\sin \theta \sin \phi, \sin \theta \cos \phi, \cos \theta)$. 
We are now in the position to give a complete description of the flow in $R^{4}$ and its relation to the flow on $S^{2}$. In doing so, we have to keep in mind that our coordinate system does not cover the poles of $S^{2}$ which correspond to the 2planes $\left(x_{1}=y_{1}=0\right)$ and $\left(x_{2}=y_{2}=0\right)$. $R^{4}$ is decomposed into concentric 3-spheres of radius $2 J^{1 / 2}$, each of which is invariant under the flow. The flow on each such sphere is completely determined by the flow on $S^{2}$ governed by the last pair of the Equations (19) (where we stretch the time by $J^{n-1}$ ). The orbits on $S^{2}$ are simply the level curves of $K^{(n)}(\boldsymbol{x})$. To the critical points on $S^{2}$ which (outside the poles) are characterized by

$$
\partial K^{(n)} / \partial \theta(\boldsymbol{e})=0, \quad \partial K^{(n)} / \partial \phi(\boldsymbol{e})=0,
$$

and whose index coincides with the sign of the determinant

$$
\left|\begin{array}{cc}
\partial^{2} K^{(n)} / \partial \theta^{2} & \partial^{2} K^{(n)} / \partial \phi \partial \theta \\
\partial^{2} K^{(n)} / \partial \phi \partial \theta & \partial^{2} K^{(n)} / \partial \phi^{2}
\end{array}\right|
$$

there corresponds a one-parametric family of periodic solutions with period

$$
1+\frac{1}{2} n J^{n-1} K^{(n)}(e) .
$$

A particular case occurs if $K^{(n)}$ is independent of $\phi$, i.e., a linear combination of the first $n+1$ Lengendre polynomials [compare formula (4)]. In this case $\theta$ is an integral, i.e., the orbits on the unit sphere in $\boldsymbol{M}$-space $S^{2}$ are circles of fixed latitude and the corresponding motion in $R^{4}$ is quasi-periodic with frequencies and

$$
\left.\begin{array}{l}
1+\frac{1}{2} J^{n-1}\left[n K^{(n)}+\cot \theta d K^{(n)} / d \theta\right] \\
\left(J^{n-1} / \sin \theta\right) d K^{(n)} / d \theta,
\end{array}\right\}
$$

respectively. The only critical points (c.p.'s) on $S^{2}$ are the poles. They represent two one-parametric families of stable periodic solutions with period

$$
1+\frac{1}{2} J^{n-1} n K^{(n)}\left( \pm e_{3}\right) \text {. }
$$

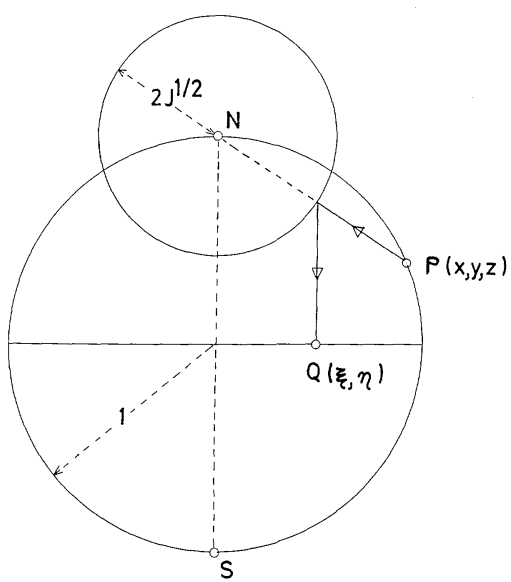

Fig. 1. The point $P$ on the unit sphere is taken into the point $Q$ by the map (25) 
The validity of the last statement can be checked using any coordinates that are regular on the poles. Such coordinates will also be used in the next section in which we will discuss the effect of the inclusion of the term $\mathcal{O}_{2 n+1}$ (compare 1.12) on the flow discussed in the present section.

In order to prepare ourselves for such a discussion we will introduce new coordinates by a variant of the stereographic projection. From the north pole of the unit sphere $S^{2}$ we first project $S^{2}$ onto a sphere of radius $2 J^{1 / 2}$ centered at the north pole and subsequently project the latter sphere vertically onto the equatorial plane (see Fig. 1).

The coordinates of the image point are denoted by $(\xi, \eta)$. We have

$$
\begin{aligned}
& \xi=(2 J)^{1 / 2} x(1-z)^{-1 / 2}=(2 J(1+z))^{1 / 2} \sin \phi \\
& \eta=(2 J)^{1 / 2} y(1-z)^{-1 / 2}=(2 J(1+z))^{1 / 2} \cos \phi .
\end{aligned}
$$

Using $L=2 J$ and $\alpha_{1}$ as coordinates in addition to $\xi$ and $\eta$, the symplectic 2 -form is

$$
\omega=d L \wedge d \alpha_{1}+d \eta \wedge d \xi
$$

and the Hamiltonian becomes

$$
H_{0}=L+(L / 2)^{n} F(\hat{\xi}, \hat{\eta})
$$

where

$$
F(\xi, \eta)=K^{(n)}\left(\left[1-\left(\xi^{2}+\eta^{2}\right) / 4\right]^{1 / 2} \xi,\left[1-\left(\xi^{2}+\eta^{2}\right) / 4\right]^{1 / 2} \eta,-1+\left(\xi^{2}+\eta^{2}\right) / 2\right)
$$

and

$$
\hat{\xi}=(2 / L)^{1 / 2} \xi, \quad \hat{\eta}=(2 / L)^{1 / 2} \eta .
$$

The validity of the statement made about the south pole of $S^{2}$ before formula (24) is now obvious, because if $K^{(n)}$ is a linear combination of the first $n+1$ Legendre polynomials, it is a polynomial in $z$ alone and therefore a polynomial $P$ in $\varrho=$ $\left(\xi^{2}+\eta^{2}\right) / L: K^{(n)}=P(\varrho)$. The corresponding Hamiltonian equations are

$$
\begin{aligned}
\dot{\xi} & =(L / 2)^{n-1} P^{\prime} \eta \\
\dot{\eta} & =-(L / 2)^{n-1} P^{\prime} \xi \\
\dot{\alpha}_{1} & =1+\frac{1}{2}(L / 2)^{n-1}\left[n P-\varrho P^{\prime}\right] .
\end{aligned}
$$

We see again that the motion in general is quasi-periodic with periods

$$
(L / 2)^{n-1} P^{\prime} \quad \text { and } \quad 1+\frac{1}{2}(L / 2)^{n-1}\left[n P-\varrho P^{\prime}\right] .
$$

However, using these new coordinates it is immediately evident that the south pole represents a stable periodic solution with frequency

$$
1+\frac{1}{2}(L / 2)^{n-1} n P(0) .
$$

The statement about the significance of the north pole contained in (24) can be proved in a similar fashion. 
In concluding this section we state the relation between the new coordinates $L, \alpha_{1}, \xi, \eta$ and the original variables of $R^{4}$ as:

$$
\begin{aligned}
& x_{1}=\left(2 L-\left(\xi^{2}+\eta^{2}\right)\right)^{1 / 2} \sin \alpha_{1} \\
& y_{1}=\left(2 L-\left(\xi^{2}+\eta^{2}\right)\right)^{1 / 2} \cos \alpha_{1} \\
& x_{2}=\xi \cos \alpha_{1}+\eta \sin \alpha_{1} \\
& y_{2}=-\xi \sin \alpha_{1}+\eta \cos \alpha_{1} .
\end{aligned}
$$

\section{The Flow of the Full Hamiltonian}

In this section we will study the effect of the inclusion of the term $\mathcal{O}_{2 n+1}$ (see 1.12), which we call perturbation term. The unperturbed motion has been studied in the last section. We have found that to each stable/unstable c.p. that the unperturbed motion possesses on $S^{2}$, there corresponds a one-parametric family of stable/unstable periodic solutions.

Furthermore, we have studied the case in which the unperturbed Hamiltonian is a linear combination of Legendre polynomials and have shown that in this case all solutions are quasi-periodic with the exception of those on the planes $x_{1}=$ $y_{1}=0$ and $x_{2}=y_{2}=0$, which are periodic.

We will show in this section that under certain additional conditions both statements essentially carry over to the flow induced in $R^{4}$ by the full Hamiltonian.

In order to give an exact formulation of the first statement, we assume that $e$ is a c.p. of the flow induced on $S^{2}$ by the unperturbed Hamiltonian. As already remarked above, we may, without loss of generality, assume that $\boldsymbol{e}=-\boldsymbol{e}_{3}$ and $A_{12}=0$ (see. 2.9), because this can always be achieved by a suitable special unitary transformation of the $z_{k}$-variables $(k=1,2)$. In the following we will therefore assume that $\boldsymbol{e}$ and $A_{12}$ have the stated values. As a consequence (2.9) will hold. Besides the partial derivatives introduced in (2.11) we will use all the other second order derivatives of $K^{(n)}$ evaluated at $\boldsymbol{x}=-\boldsymbol{e}_{3}$, which we will denote accordingly

$$
A_{33}=\left(\partial^{2} K^{(n)} / \partial z^{2}\right)_{\boldsymbol{x}=-\boldsymbol{e}_{3}}, \quad A_{23}=\left(\partial^{2} K^{(n)} / \partial y \partial z\right)_{\boldsymbol{x}=-\boldsymbol{e}_{3}}, \quad A_{13}=\left(\partial^{2} K^{(n)} / \partial x \partial z\right)_{\boldsymbol{x}=-\boldsymbol{e}_{3}} .
$$

From (2.5) we derive

$$
\lambda=-\left(\partial K^{(n)} / \partial z\right)_{\boldsymbol{x}=-\boldsymbol{e}_{3}} .
$$

In addition we use the abbreviations

$$
A=\lambda-A_{11}, \quad B=\lambda-A_{22}, \quad C=\lambda-A_{33}
$$

and

$$
D=\left|\begin{array}{ccc}
A & 0 & -A_{13} \\
0 & B & -A_{23} \\
-A_{13} & -A_{23} & C
\end{array}\right| .
$$

We are now in the position to state the first theorem. 
Theorem 1. (i) To each unstable critical point (remember c.p. means nondegenerate c.p.) of the flow that the unperturbed Hamiltonian induces on $S^{2}$ there corresponds an unstable one-parametric family of periodic solutions of the equations associated with the full Hamiltonian, the family parameter being the energy.

(ii) An analogous statement holds if the critical point is stable provided that in addition the following expression is nonzero:

$$
12\left(A^{3} A_{23}^{2}+B^{3} A_{13}^{2}\right)+4(A B)^{2}(A+B+C)-3 D(A+B)^{2},
$$

where

$$
A B=\Delta(\lambda)>0 .
$$

In order to prove the theorem, we use the coordinates introduced in the previous section. Taking (2.27) into account, the full Hamiltonian may be written in the form

$$
H=L+(L / 2)^{n} F\left((2 / L)^{1 / 2} \xi,(2 / L)^{1 / 2} \eta\right)+\mathcal{O}\left(L^{n+1 / 2}\right),
$$

where $\mathcal{O}\left(L^{n+1 / 2}\right)$ after division by $L^{n+1 / 2}$ is a function of the variables

$$
L^{1 / 2}, \quad(2 / L)^{1 / 2} \xi \quad(2 / L)^{1 / 2} \eta, \quad \alpha_{1},
$$

which is real analytic in these variables at each point $\left(0,0,0, \alpha_{1}\right)$ and periodic in $\alpha_{1}$ with period $2 \pi$.

Setting $L=\varepsilon^{2} \hat{L}, \xi=\varepsilon \hat{\xi}, \eta=\varepsilon \hat{\eta}$ [which amounts to setting $z_{k}=\varepsilon \hat{z}_{k}(k=1,2)$, we obtain

$$
\hat{H}=(L / 2)\left[2+\left(L \varepsilon^{2} / 2\right)^{n-1} F\left((2 / L)^{1 / 2} \xi,(2 / L)^{1 / 2} \eta\right)+\mathcal{O}\left(\left(\varepsilon^{2} L\right)^{n-1 / 2}\right)\right]
$$

where we have dropped the carets everywhere, except on $H$. Notice that

$$
H=\varepsilon^{2} \hat{H} .
$$

Consider now the energy surface $\hat{H}=2$, i.e., $H=2 \varepsilon^{2}$. On this surface relation (8) defines $L$ as a function of $\xi, \eta, \alpha_{1}, \varepsilon$ which is real analytic at $\left(0,0, \alpha_{1}, 0\right)$ and which for $\varepsilon=0$ is identical to two. Subtracting its value at $\left(0,0, \alpha_{1}, \varepsilon\right)$, we obtain from this function a function which we will denote by $\Lambda$ and whose power series in $\varepsilon$ begins as follows:

$$
\Lambda\left(\xi, \eta, \alpha_{1}, \varepsilon\right)=\varepsilon^{2(n-1)}[-F(\xi, \eta)+\mathcal{O}(\varepsilon)] .
$$

$\Lambda$ together with the symplectic 2 -form

$$
d \xi \wedge d \eta
$$

determines the flow on the energy surface $H=2 \varepsilon^{2}$.

If we follow the trajectories from $\alpha_{1}=0$ to $\alpha_{1}=2 \pi$ we obtain a measurepreserving map

$$
M: \begin{aligned}
& \xi_{1}=\xi+2 \pi \varepsilon^{2(n-1)} f(\xi, \eta, \varepsilon) \\
& \eta_{1}=\eta+2 \pi \varepsilon^{2(n-1)} g(\xi, \eta, \varepsilon)
\end{aligned}
$$


where

$$
\begin{aligned}
& f(\xi, \eta, 0)=F_{\eta}(\xi, \eta) \\
& g(\xi, \eta, 0)=-F_{\xi}(\xi, \eta) .
\end{aligned}
$$

(The subscripts $\xi, \eta$ denote the corresponding partial derivatives.) In the following we use the expansion

$$
\begin{aligned}
-F(\xi, \eta)= & \frac{1}{2}\left[\left(\lambda-A_{11}\right) \xi^{2}+\left(\lambda-A_{22}\right) \eta^{2}\right]+(1 / 8)\left(A_{11} \xi^{2}+A_{22} \eta^{2}\right) \\
& -\left(A_{33} / 8\right)\left(\xi^{2}+\eta^{2}\right)^{2}-\frac{1}{2}\left[A_{13} \xi+A_{23} \eta\right]\left(\xi^{2}+\eta^{2}\right)+\mathcal{O}_{5} .
\end{aligned}
$$

In particular,

$$
\begin{aligned}
& f_{\xi}(0,0,0)=g_{\eta}(0,0,0)=0, \\
& f_{\eta}(0,0,0)=F_{\eta \eta}(0,0)=\lambda-A_{22}=B \\
& g_{\xi}(0,0,0)=-F_{\xi \xi}(0,0)=-\left(\lambda-A_{11}\right)=-A .
\end{aligned}
$$

Hence,

$$
\left|\begin{array}{ll}
f_{\xi} & f_{\eta} \\
g_{\xi} & g_{\eta}
\end{array}\right|_{\xi=\eta=\varepsilon=0}=A B=\Delta(\lambda) \neq 0
$$

by assumption. Taking into account that

$$
f(0,0,0)=g(0,0,0)=0,
$$

we see that there exist two functions $\xi_{0}(\varepsilon), \eta_{0}(\varepsilon)$, real analytic at $\varepsilon=0$, such that

$$
\begin{array}{ll}
f\left(\varepsilon \xi_{0}(\varepsilon),\right. & \left.\varepsilon \eta_{0}(\varepsilon), \varepsilon\right)=0 \\
g\left(\varepsilon \xi_{0}(\varepsilon),\right. & \left.\varepsilon \eta_{0}(\varepsilon), \varepsilon\right)=0 .
\end{array}
$$

Clearly $\left(\varepsilon \xi_{0}(\varepsilon), \varepsilon \eta_{0}(\varepsilon)\right)$ is a fixed point of the map $M$. Thus, we have shown that to each c.p. of the unperturbed Hamiltonian there exists a one-parametric family of periodic solutions of the full Hamiltonian.

Let $\varepsilon \xi_{0}\left(\alpha_{1}, \varepsilon\right), \varepsilon \eta_{0}\left(\alpha_{1}, \varepsilon\right)$ be the periodic solutions corresponding to the Hamiltonian (10) found in this way. We set

$$
\begin{aligned}
& \hat{\xi}=\xi-\varepsilon \xi_{0}\left(\alpha_{1}, \varepsilon\right) \\
& \hat{\eta}=\eta-\varepsilon \eta_{0}\left(\alpha_{1}, \varepsilon\right) .
\end{aligned}
$$

The new Hamiltonian is

$$
\begin{aligned}
\hat{\Lambda}\left(\hat{\xi}, \hat{\eta}, \alpha_{1}, \varepsilon\right) & =\Lambda\left(\hat{\xi}+\varepsilon \xi_{0}, \hat{\eta}+\varepsilon \eta_{0}, \alpha_{1}, \varepsilon\right)+\varepsilon\left(\hat{\xi} \eta_{0}^{\prime}-\hat{\eta} \xi_{0}^{\prime}\right) \\
& =\varepsilon^{2(n-1)}[-F(\hat{\xi}, \hat{\eta})+\mathcal{O}(\varepsilon)] .
\end{aligned}
$$

Dropping the carets, we recognize that the coordinates $\xi, \eta$ can be chosen in such a way that on every energy surface $H=2 \varepsilon^{2}$ the periodic solution is simply given by $\xi=\eta=0 . \Lambda\left(\xi, \eta, \alpha_{1}, \varepsilon\right)$ therefore begins with a quadratic expression whose coefficients depend on $\alpha_{1}, \varepsilon$. From the expansion (14) we see that for $\varepsilon=0$ the quadratic part of $\Lambda$ is definite or indefinite depending on whether $\Delta(\lambda)>0$ or $\Delta(\lambda)<0$. But because $\Lambda$ is periodic in $\alpha_{1}$, this property prevails for all $\alpha_{1}$ and for all sufficiently small $|\varepsilon|$. If $\Delta(\lambda)<0$, it follows immediately that the map $M$ and therefore the 
corresponding family of periodic solutions is unstable. Let us therefore concentrate on the case $\Delta(\lambda)>0$. We introduce normal coordinates $r, \gamma$ such that for sufficiently small $|\varepsilon|$ the Hamiltonian $\Lambda$ in these variables has an expansion

$$
\Lambda\left(r, \gamma, \alpha_{1}, \varepsilon\right)=\frac{1}{2}\left[v\left(\alpha_{1}, \varepsilon\right) r^{2}-\frac{1}{2} \kappa\left(\alpha_{1}, \varepsilon\right) r^{4}+\mathcal{O}_{5}\right]
$$

where $\mathcal{O}_{5}$ is a function periodic in $\alpha_{1}, \gamma$ with period $2 \pi$ and real analytic in its variables at $r=0, \alpha_{1}, \gamma, \varepsilon=0$. The power series expansion of this function begins with a term of order five in $r$. Taking into account that the fundamental symplectic two-form in the new variables is

$$
r d r \wedge d \gamma,
$$

the equations corresponding to (19) become

$$
\begin{aligned}
& \dot{\gamma}=\varepsilon^{2(n-1)}\left[v-\kappa r^{2}\right]+\mathcal{O}_{3} \\
& \dot{r}=\varepsilon^{2(n-1)} \mathcal{O}_{4} .
\end{aligned}
$$

Hence, we see that the map $M$ in these coordinates has the form of a perturbed twist:

$$
\begin{aligned}
& r_{1}=r+\varepsilon^{2(n-1)} \mathcal{O}_{4} \\
& \gamma_{1}=\gamma+\varepsilon^{2(n-1)}\left(\nu^{*}-\kappa^{*} r^{2}\right)+\mathcal{O}_{3}
\end{aligned}
$$

which, moreover, preserves the area element (20). In (21) $v^{*}, \kappa^{*}$ denote the functions of $\varepsilon$ obtained by averaging the functions $v, \kappa$ over $\alpha_{1}$; also, the perturbation terms are no longer functions of $\alpha_{1}$. Dropping them we are left with a twist $M_{0}$ that is exactly nondegenerate if $\kappa^{*}(\varepsilon) \neq 0$. A straightforward but somewhat lengthy calculation yields

$$
\begin{aligned}
v\left(\alpha_{1}, 0\right) & =v^{*}(0)=(\Delta(\lambda))^{1 / 2} \\
\kappa^{*}(0) & =(1 / 2 \Delta(\lambda)) \quad \text { expression }(5) .
\end{aligned}
$$

It follows that under the conditions of the theorem the twist $M_{0}$ is nondegenerate for sufficiently small $|\varepsilon|$.

We are now ready to show that the fixed point $r=0, \varepsilon=\varepsilon_{0} \neq 0$ (sufficiently small $\varepsilon_{0}$ ) is stable. This will imply orbital stability of the periodic solution on the energy surface $H=2 \varepsilon_{0}^{2}$.

To each small positive number $\delta$ select a "strongly irrational" number $\omega_{\delta}$ in the interval

$$
\left(\varepsilon_{0}^{2(n-1)}\left[\nu^{*}\left(\varepsilon_{0}\right)-\delta^{2}\left|\kappa^{*}\left(\varepsilon_{0}\right)\right|\right], \varepsilon_{0}^{2(n-1)} \nu^{*}\left(\varepsilon_{0}\right)\right) .
$$

This is possible because the "strongly irrational" numbers, i.e., numbers $\omega$ satisfying the finitely many inequalities

$$
|\omega q-p| \geqq c_{0} / q^{\mu} \quad(p, q=1,2, \ldots)
$$

with some $c_{0}>0$ and some $\mu \geqq 2$, are dense on the real axis (Ref. [4], p. 191). There exists an open interval $I_{\delta}$ about $\varepsilon_{0}$, not containing $\varepsilon=0$, such that for all $\varepsilon \in I_{\delta}, \omega_{\delta}$ is also in the interval with the same endpoints as (22), except that $\varepsilon_{0}$ is replaced by $\varepsilon$. 
According to Moser's twist theorem (Ref. $[3,4,5]$ ) to each $\varepsilon \in I$, there exists an analytic curve $r=r_{\delta}(\gamma, \varepsilon)$ which by the map $M$ is mapped into itself via the transformation

$$
\gamma \rightarrow \gamma+\omega_{\delta} .
$$

Because this curve is obtained by a continuous deformation of the circle of radius

$$
\left[v^{*}(\varepsilon)-\omega_{\delta} / \varepsilon^{2(n-1)}\right]^{1 / 2}\left|\kappa^{*}(\varepsilon)\right|^{-1 / 2},
$$

and this number lies in the interval $(0, \delta)$ by (22), the inequality $r_{\delta}(\gamma, \varepsilon)<2 \delta$ is valid for $\varepsilon \in I_{\delta}$ and for sufficiently small $\delta$. If $U$ is a preassigned neighborhood of the point $r=0, \varepsilon=\varepsilon_{0}$, then the neighborhood

$$
\left\{r, \gamma, \varepsilon \mid r<r_{\delta}(\gamma, \varepsilon), \varepsilon \in I_{\delta}\right\}
$$

for sufficiently small $\delta$ is contained in $U$ and invariant under $M$, proving the stability of the map $M$ and, therefore, our theorem.

We turn now to an exact statement and proof of our second contention made at the beginning of this section.

Theorem 2. If $K^{(n)}$ is a polynomial of degree at least 2 of the variable $z$ alone, then for sufficiently small $\varepsilon$ the energy surface $H=2 \varepsilon^{2}$ (with $H$ as defined in 1.12) is filled with invariant tori except for a portion whose measure is small with $\varepsilon$. form

Proof. The conditions of the theorem imply that $\Lambda$ as defined in (10) has the

$$
\Lambda\left(\xi, \eta, \alpha_{1}, \varepsilon\right)=\varepsilon^{2(n-1)}[-P(\varrho)+\mathcal{O}(\varepsilon)],
$$

where $P$ has been defined in (2.29) and

$$
\varrho=\left(\xi^{2}+\eta^{2}\right) / 2 \text {. }
$$

Setting $\xi=R \sin \phi, \eta=R \cos \phi$, the Hamiltonian becomes a function $\tilde{\Lambda}$ periodic in $\phi, \alpha_{1}$ with period $2 \pi$ and real analytic in its variables at $R=0, \phi, \alpha_{1}, \varepsilon=0$, whose power series expansion begins as follows:

$$
\tilde{\Lambda}\left(R, \phi, \alpha_{1}, \varepsilon\right)=\varepsilon^{2(n-1)}\left[-P\left(\varrho^{2} / 2\right)+\mathcal{O}(\varepsilon)\right] .
$$

$\tilde{\Lambda}$ together with the symplectic two-form $-R d R \wedge d \phi$ governs the flow on the energy surface $H=2 \varepsilon^{2}$. The corresponding Hamilton equations therefore are

$$
\begin{aligned}
& \dot{\phi}=\varepsilon^{2(n-1)}\left[P^{\prime}\left(R^{2} / 2\right)+\mathcal{O}(\varepsilon)\right] \\
& \dot{R}=\mathcal{O}\left(\varepsilon^{2(n-1)}\right) .
\end{aligned}
$$

Thus, under the present assumptions also, the map $M$ defined above will assume the form of an analytically perturbed, nondegenerate twist

$$
\begin{aligned}
& R_{1}=R+\mathcal{O}\left(\varepsilon^{2(n-1)}\right) \\
& \phi_{1}=\phi+\varepsilon^{2(n-1)} 2 \pi P^{\prime}\left(R^{2} / 2\right)+\mathcal{O}\left(\varepsilon^{2 n-1}\right) .
\end{aligned}
$$

Our Theorem 2 follows in a standard manner from Moser's twist theorem. 


\section{The case $n=2$}

We specialize to the case $n=2$ and write $K_{2}$ in the form

$$
K_{2}=\frac{1}{2} a_{0} J^{2}+(\boldsymbol{b}, \boldsymbol{M})+\frac{1}{2}(\boldsymbol{M}, \mathfrak{U} \boldsymbol{M}),
$$

where $(\boldsymbol{a}, \boldsymbol{b})=\sum_{i=1}^{3} a_{i} b_{i}$ is the usual inner product of $R^{3}$ and $\mathfrak{A}$ is a real symmetric matrix

$$
(\mathfrak{U} M)_{k}=\sum_{j=1}^{3} a_{k j} M_{j} \quad(k=1,2,3) .
$$

Clearly, $a_{0}, a_{11}, a_{22}, a_{33}$ are not uniquely determined, but a substitution

$$
a_{0} \rightarrow a_{0}-\mu, \quad a_{k k} \rightarrow a_{k k}+\mu \quad(k=1,2,3)
$$

leaves (1) invariant [compare (1.14)]. Moreover, a transformation of the $z$-variables as described in (1.15) replaces $\boldsymbol{b}, \mathfrak{U}$ by

$$
\hat{\boldsymbol{b}}=R \boldsymbol{b}, \quad \hat{\mathfrak{U}}=R \mathfrak{U} R^{T},
$$

where $R$ is the matrix defined in (1.17) and $R^{T}$ its transposition. $U$ in (1.15) may therefore be chosen in such a way that $\hat{\mathfrak{A}}$ is a diagonal matrix with the eigenvalues $a_{1}, a_{2}, a_{3}$ of $\mathfrak{A}$ along the diagonal. Of course, a transformation of type (2), carried out in the matrix $\mathfrak{A}$, will also replace the eigenvalue $a_{k}$ by $a_{k}+\mu$, such that its effect on $\hat{\mathfrak{U}}$ is still described by (3) with $a_{k k}$ replaced by $a_{k}(k=1,2,3)$.

In the following we will assume that the matrix $\mathfrak{A}$ has been diagonalized and that the eigenvalues are mutually distinct. The case of degenerate eigenvalues will be dealt with later. In any case, the assumption that $\mathfrak{U}$ is diagonal implies that the eigenbasis of $\mathfrak{A}$ coincides with the standard frame $\boldsymbol{e}_{1}, \boldsymbol{e}_{2}, \boldsymbol{e}_{3}$ of $R^{3}$. In order to find all families of periodic solutions connected with Hamiltonian (1.12) (where now $n=2$ ), we have to determine all c.p.'s together with their indices that the function

$$
K^{(2)}(x)=a_{0} / 2+b_{1} x+b_{2} y+b_{3} z+\frac{1}{2}\left(a_{1} x^{2}+a_{2} y^{2}+a_{3} z^{2}\right)
$$

possesses on the unit sphere $S^{2}$.

As explained in Section 1, the c.p.'s are the endpoints of those unit vectors $\boldsymbol{e}=\left(e_{1}, e_{2}, e_{3}\right)$ to which a real number $\lambda$ can be found with the property

$$
\left(\lambda-a_{i}\right) e_{i}=b_{i}
$$

for $i=1,2,3$. In our classification scheme of possible c.p.'s the function

$$
g(\lambda)=1-b_{1}^{2} /\left(\lambda-a_{1}\right)^{2}-b_{2}^{2} /\left(\lambda-a_{2}\right)^{2}-b_{3}^{2} /\left(\lambda-a_{3}\right)^{2}
$$

will play a central role. Assuming that $b_{1} b_{2} b_{3} \neq 0$, every c.p. will be the endpoint of a vector

$$
\boldsymbol{e}\left(\lambda_{0}\right)=\left(b_{1} /\left(\lambda_{0}-a_{1}\right), b_{2} /\left(\lambda_{0}-a_{2}\right), b_{3} /\left(\lambda_{0}-a_{3}\right)\right)
$$

where $\lambda_{0}$ is a zero of $g$. 
We shall call c.p.'s that arise in this manner from zeros of $g$ "c.p.'s of the first kind". C.p.'s of a different nature arise if $b_{k}=0$ and $g\left(a_{k}\right)>0(k=1,2,3)$. If, e.g., $b_{1}=0$ and $g\left(a_{1}\right)>0$, then

$$
\boldsymbol{f}_{1}^{ \pm}=\left( \pm\left[g\left(a_{1}\right)\right]^{1 / 2}, b_{2} /\left(a_{1}-a_{2}\right), b_{3} /\left(a_{1}-a_{3}\right)\right)
$$

is a pair of unit vectors satisfying (6) with $\lambda=a_{1}$. It consists of position vectors of c.p.'s that we shall call "c.p.'s of the second kind". To give a more geometrical description of the conditions under which c.p.'s of the second kind are present, let us momentarily assume that the matrix $\mathfrak{A}$ is in general form. If its eigenvalues are all distinct and if $\boldsymbol{b}$ is orthogonal to an eigendirection with corresponding eigenvalue $\lambda$, then the equation

$$
(\lambda-\mathfrak{U}) \boldsymbol{e}=\boldsymbol{b}
$$

[which replaces (6) under this more general assumptions] is satisfied by a oneparametric family of vectors whose endpoints form a straight line. This family contains either two, one or no unit vectors, depending on whether the line intersects, touches or does not intersect the unit sphere.

If $\mathfrak{U}$ is diagonal and $b_{1}=0$, then $\lambda=a_{1}$, and $g\left(a_{1}\right)>0$ is precisely the condition under which the line of solutions of (6) intersects the unit sphere. The position vectors of the points of intersection are given in (9).

What are the indices of these critical points? Applying the criterion expressed in connection with formula (2.7) to the special case $K^{(2)}$, we obtain the following formula:

$$
\text { ind } e=\operatorname{sgn} \Delta(\lambda),
$$

where $\Delta(\lambda)$ is the function

$$
\Delta(\lambda)=\left(\lambda-a_{2}\right)\left(\lambda-a_{3}\right) e_{1}^{2}+\left(\lambda-a_{3}\right)\left(\lambda-a_{1}\right) e_{2}^{2}+\left(\lambda-a_{1}\right)\left(\lambda-a_{2}\right) e_{3}^{2} .
$$

This criterion, when applied to the members of the pair of c.p.'s (we identify each c.p. with its position vector), yields the following result:

$$
\text { ind } f_{k}^{ \pm}=\operatorname{sgn} \prod_{\substack{j=1 \\ j \neq k}}^{3}\left(a_{k}-a_{j}\right) \quad(k=1,2,3) \text {. }
$$

In order to obtain a suitable criterion for the indices of a c.p. of the first kind, we observe that an alternate expression for $\Delta(\lambda)$ is

$$
\Delta(\lambda)=\frac{1}{2}\left(\lambda-a_{1}\right)\left(\lambda-a_{2}\right)\left(\lambda-a_{3}\right) g^{\prime}(\lambda) .
$$

Hence, the index of the c.p. $e\left(\lambda_{0}\right)$ is determined by the position of the zero $\lambda_{0}$ of $g(\lambda)$ relative to $a_{1}, a_{2}, a_{3}$ and the sign of the slope of the graph of $g$ at $\lambda_{0}$.

Let us pause in order to study in detail how a pair of c.p.'s of the second kind emerges. For that purpose we will assume that the eigenvalues $a_{1}, a_{2}, a_{3}$ are distinct, that $b_{1}=0$ and that $b_{2}, b_{3}$ are real analytic functions of some parameter $\mu$ at $\mu=0$. We could also assume that the $a_{k}$ 's are function of $\mu$ as is the case, for 
example, in the Hénon-Heiles Hamiltonian. However, in order to keep the argument as simple as possible, we keep the $a_{k}$ 's constant.

$$
g(\lambda, \mu)=1-b_{2}^{2}(\mu) /\left(\lambda-a_{2}\right)^{2}-b_{3}^{2}(\mu) /\left(\lambda-a_{3}\right)^{2}
$$

is an analytic function of its variables for all $\lambda \neq a_{2}, a_{3}$ and in a neighborhood of $\mu=0$. We will assume that it has the following properties:

$$
g\left(a_{1}, 0\right)=0, \quad g_{\mu}\left(a_{1}, 0\right)>0, \quad g^{\prime}\left(a_{1}, 0\right) \neq 0,
$$

where the subscript $\mu$ denotes partial derivative with respect to $\mu$ and the prime partial derivative with respect to $\lambda$. Under our assumptions, there exists a function $\lambda(\mu)$, real analytic at $\mu=0$ with the properties

$$
\begin{aligned}
& g(\lambda(\mu), \mu)=0, \quad \lambda(0)=a_{1}, \quad \operatorname{sgn} g^{\prime}(\lambda(\mu), \mu)=\operatorname{sgn} g^{\prime}\left(a_{1}, 0\right) \\
& \operatorname{sgn} \lambda^{\prime}(\mu)=\operatorname{sgn} \lambda^{\prime}\left(a_{1}\right)=-\operatorname{sgn} g^{\prime}\left(a_{1}, 0\right),
\end{aligned}
$$

provided $|\mu|$ is sufficiently small.

Hence, according to (13)

$$
\text { ind } \begin{aligned}
e(\lambda(\mu)) & =\operatorname{sgn}\left(\lambda(\mu)-a_{1}\right)\left(\lambda(\mu)-a_{2}\right)\left(\lambda(\mu)-a_{3}\right) g^{\prime}(\lambda(\mu), \mu) \\
& =\operatorname{sgn}\left[\mu \cdot \lambda^{\prime}\left(a_{1}\right)\left(a_{1}-a_{2}\right)\left(a_{1}-a_{3}\right) g^{\prime}\left(a_{1}, 0\right)\right] \\
& =-\varepsilon \operatorname{sgn} \mu, \quad \text { where } \varepsilon=\operatorname{sgn}\left[\left(a_{1}-a_{2}\right)\left(a_{1}-a_{3}\right)\right] .
\end{aligned}
$$

As long as $\mu<0$, ind $\boldsymbol{e}(\lambda)=\varepsilon$. For $\mu=0, \boldsymbol{f}_{1}^{ \pm}$, and $\boldsymbol{e}\left(a_{1}\right)$ all agree and are given by the vector

$$
\left(0, b_{2}(0) /\left(a_{1}-a_{2}\right), b_{3}(0) /\left(a_{1}-a_{3}\right)\right) .
$$

By a continuity argument this c.p. still has index $\varepsilon$. As soon as $\mu>0$ the pair $f_{1}^{ \pm}$ with total index $2 \varepsilon$ [see (12)] splits off from $e(\lambda(\mu))$ and the latter c.p. simultaneously changes its index from $\varepsilon$ to $-\varepsilon$. This process could be represented symbolically by

$$
\boldsymbol{e}(\varepsilon) \rightarrow \boldsymbol{e}(-\varepsilon)+\boldsymbol{f}_{1}^{ \pm}(2 \varepsilon) .
$$

We proceed now to discuss the classification scheme of c.p.'s on $S^{2}$. First, we will assume that all eigenvalues $a_{1}, a_{2}, a_{3}$ are distinct and only later turn to the case in which two of them coincide. In the case $a_{1}=a_{2}=a_{3}$ a transformation of type (3) will make all eigenvalues zero and a transformation of type (1.15) will rotate the vector $\boldsymbol{b}$ into the 3-direction: the motion is a pure rotation about the 3-axis and accordingly the only two c.p.'s are the poles. Obviously they are stable.

Until further notice, we will now assume that the $a_{k}$ 's are mutually distinct. The various cases we will distinguish differ by the number of components of the $\boldsymbol{b}$-vector that vanish.

We state in advance that in all these various cases (omitting degeneracies) there exist only three possibilities with regard to the number of c.p.'s on $S^{2}$ and their indices. On $S^{2}$ the function (5) has either:

(1) 2 elliptic

(2) 3 elliptic and 1 hyperbolic

(3) 4 elliptic and 2 hyperbolic 
c.p.'s. This is in agreement with the general fact that the total index of a continuous vector field on $S^{2}$ is equal to two.

Case 0 . The vector $\boldsymbol{b}$ has no vanishing components.

Let $I_{1}, I_{2}$ be the two adjacent bounded open intervals whose endpoints are the $a_{k}$ 's $(k=1,2,3)$, and let $I_{0}$ be the complement in $R$ of the closure of their union. Drawing the graph of $g(\lambda)$ shows: $g$ has always two real zeros $\lambda_{0}^{ \pm}$in $I_{0}$, to which, according to the criterion (13), there corresponds an elliptic pair of c.p.'s. Furthermore, $g$ has exactly one relative maximum $G_{k}$ in each of the intervals $I_{k}(k=1,2)$. If $G_{k}<0(k=1,2)$, there are no other c.p.'s and possibility (1) is realized. If $G_{1} G_{2}<0, g$ has another pair of real zeros either in $I_{1}$ or in $I_{2}$. The zero closer to the common endpoint of $I_{1}$ and $I_{2}$ gives rise to a hyperbolic c.p., the other one to an elliptic c.p.; i.e., possibility (2) is realized. If finally $G_{k}>0(k=1,2)$, all six real zeros mentioned so far are present, showing that possibility (3) is realized.

Case 1. The vector $\boldsymbol{b}$ has exactly one vanishing component. We will treat the case $b_{1} b_{2} \neq 0, b_{3}=0$. The cases $b_{2} b_{3} \neq 0, b_{1}=0$ and $b_{3} b_{1} \neq 0, b_{2}=0$ are obtained from this case by a cyclic permutation of the subscripts. By a transformation of type (1.15) we could also reduce the latter two cases to the first.

Let $I$ be the open interval with endpoints $a_{1}, a_{2}$, and $I_{0}$ the interior of its complement in $R . g$ has a unique relative maximum in $I$, given by

$$
G=g\left(\left(b_{1}^{2 / 3} a_{2}+b_{2}^{2 / 3} a_{1}\right) /\left(b_{1}^{2 / 3}+b_{2}^{2 / 3}\right)\right) .
$$

Let $G \neq 0$ and $\varrho=\operatorname{sgn} G$. $g$ always has two real zeros in $I_{0}$ which we denote by $\lambda_{0}^{ \pm}$, and another pair $\lambda^{ \pm}$occurs in $I$, if and only if $\varrho=1$. Of the pair $\lambda_{0}^{ \pm}$(or $\lambda^{ \pm}$) let $\lambda_{0}^{-}$(or $\lambda^{-}$) be the one closer to $a_{3}$. If and only if $g\left(a_{3}\right)>0$, the pair of c.p.'s of the second kind $f_{3}^{ \pm}$is present. The indices of the various c.p.'s depend on whether $a_{3}$ belongs to $I$ or $I_{0}$.

(a): $a_{3} \in I_{0}$. We have

$$
\begin{aligned}
\text { ind } \boldsymbol{e}\left(\lambda_{0}^{+}\right) & =1, \quad \text { ind } e\left(\lambda_{0}^{-}\right)=-\varepsilon, \quad \text { ind } e\left(\lambda^{ \pm}\right)= \pm 1 \\
\text { ind } \boldsymbol{f}_{3}^{ \pm} & =1, \quad \text { where } \varepsilon=1 \text { for } g\left(a_{3}\right)>0 \text { and } \varepsilon=-1 \text { otherwise. }
\end{aligned}
$$

(b): $a_{3} \in I$. ind $e\left(\lambda_{0}^{ \pm}\right)=1$, ind $e\left(\lambda^{+}\right)=1$, ind $e\left(\lambda^{-}\right)=\varepsilon$, ind $\boldsymbol{f}_{3}^{ \pm}=-1$.

In any case, of the three possibilities listed in (14), the one with the number

$$
1+(1+\varepsilon) / 2+(1+\varrho) / 2
$$

is present.

Case 2. Exactly two components of the vector $\boldsymbol{b}$ vanish. We shall assume $b_{3} \neq 0, b_{1}=b_{2}=0$. The other two cases $\left(b_{1} \neq 0, b_{2}=b_{3}=0\right.$, and $\left.b_{2} \neq 0, b_{3}=b_{1}=0\right)$ can either be obtained from the first by a cyclic permutation of the subscripts, or they can be reduced to it by a transformation of type (1.15). Because $g$ has just two zeros, namely:

$$
\lambda^{ \pm}=a_{3} \pm b_{3}
$$

we can treat this case more explicitly than the previous ones. Notice that to the two roots $\lambda^{ \pm}$there correspond the north and the south pole of $S^{2}$. We first assume 
that the numbers

$$
\alpha_{r}^{ \pm}=a_{3}-a_{r} \pm b_{3} \quad(r=1,2)
$$

are non-zero and set

$$
\begin{aligned}
\varepsilon_{r}^{ \pm} & =\operatorname{sgn} \alpha_{r}^{ \pm} \quad(r=1,2) \\
\varepsilon_{3} & =\operatorname{sgn} b_{3} .
\end{aligned}
$$

From (10) and (11) we find

$$
\text { ind } \boldsymbol{e}\left(\lambda^{ \pm}\right)=\varepsilon_{1}^{ \pm} \varepsilon_{2}^{ \pm}, \quad \text { where } \quad \boldsymbol{e}\left(\lambda^{ \pm}\right)= \pm \boldsymbol{e}_{3} .
$$

Because $\alpha_{r}^{+}-\alpha_{r}^{-}=2 b_{3}$, only the two cases

$$
\varepsilon_{r}^{ \pm}= \pm \varepsilon_{3} \quad \text { and } \quad \varepsilon_{r}^{+}=\varepsilon_{r}^{-} \equiv \varepsilon_{r} \quad(r=1,2)
$$

can occur, and we easily check that the first case corresponds to $g\left(a_{r}\right)<0$, the second one to $g\left(a_{r}\right)>0(r=1,2)$. In the second case the pair of c.p.'s $\boldsymbol{f}_{r}^{ \pm}(r=1,2)$ is present. The indices of these c.p.'s of the second kind are given according to (12) by the formulae

$$
\begin{aligned}
& \text { ind } f_{1}^{ \pm}=\varepsilon_{1} \operatorname{sgn}\left(a_{2}-a_{1}\right)=\operatorname{sgn}\left[\left(\alpha_{1}^{ \pm}\right)^{2}-\alpha_{1}^{ \pm} \alpha_{2}^{ \pm}\right] \\
& \text {ind } f_{2}^{ \pm}=\varepsilon_{2} \operatorname{sgn}\left(a_{1}-a_{2}\right)=\operatorname{sgn}\left[\left(\alpha_{2}^{ \pm}\right)^{2}-\alpha_{1}^{ \pm} \alpha_{2}^{ \pm}\right] .
\end{aligned}
$$

Here the last expression on each line tells us:

If at least one of the c.p.'s of the first kind has an index of -1 and if $\varepsilon_{r}^{+}=\varepsilon_{r}^{-}$, then the c.p.'s $\boldsymbol{f}_{r}^{ \pm}$are both elliptic. $(r=1,2$.)

We are now ready to distinguish different subcases

(a) $\varepsilon_{1}^{ \pm}= \pm \varepsilon_{3}, \varepsilon_{2}^{ \pm}= \pm \varepsilon_{3}$ : North and south pole are the only c.p.'s and both are elliptic.

(b) $\varepsilon_{1}^{+}=\varepsilon_{1}^{-} \equiv \varepsilon_{1}, \varepsilon_{2}^{ \pm}= \pm \varepsilon_{3}$ :

ind $\left( \pm \boldsymbol{e}_{3}\right)=\varepsilon_{1} \varepsilon_{2}, \boldsymbol{f}_{1}^{ \pm}$exists and has an index of +1 .

(c) Subscripts 1 and 2 interchanged in (b).

(d) $\varepsilon_{1}^{+}=\varepsilon_{1}^{-} \equiv \varepsilon_{1}, \varepsilon_{2}^{+}=\varepsilon_{2}^{-} \equiv \varepsilon_{2}$ :

ind $\left( \pm e_{3}\right)=\varepsilon_{1} \varepsilon_{2}$. Both pairs $f_{1}^{ \pm}, f_{2}^{ \pm}$are present with index:

$$
\begin{aligned}
& \text { ind } \boldsymbol{f}_{1}^{ \pm}=\text {ind } \boldsymbol{f}_{2}^{ \pm}=1, \text { if } \varepsilon_{1}=-\varepsilon_{2}, \\
& \text { ind } \boldsymbol{f}_{1}^{ \pm}=\varepsilon_{1} \operatorname{sgn}\left(a_{2}-a_{1}\right)=- \text { ind } \boldsymbol{f}_{2}^{ \pm}, \text {if } \varepsilon_{1}=\varepsilon_{2} .
\end{aligned}
$$

Finally, we observe that whenever one of the numbers $\alpha_{r}^{+}, \alpha_{r}^{-}$vanishes, the other does not, and although $g\left(a_{r}\right)=0$, we infer from the study of how a pair of c.p.'s of the second kind breaks away from one of the first kind (p. 30) that the situation is essentially the same as for $g\left(a_{r}\right)<0$, i.e., we have to set $\varepsilon_{r}^{ \pm}= \pm \varepsilon_{3}$.

We illustrate the present case by taking up the example of Hénon-Heiles (Ref. $[1,6]$ ). The Hamiltonian is

$$
H=\frac{1}{2}\left(x_{1}^{2}+y_{1}^{2}\right)+\frac{1}{2}\left(x_{2}^{2}+y_{2}^{2}\right)+x_{1}^{2} x_{2}+\mu x_{3}^{2} .
$$


It has (diagonalized) Gustavson normal form (5), where

$$
\begin{array}{ll}
a_{0}=-\frac{1}{2}\left[13 / 3+12 \mu+15 \mu^{2}\right] ; & a_{1}=-a_{2}=2-\mu \\
a_{3}=\frac{1}{2}\left[-15 \mu^{2}+12 \mu+1\right] ; & b_{1}=b_{2}=0 \\
b_{3}=(15 / 2)\left(1 / 9-\mu^{2}\right) . &
\end{array}
$$

In our classification scheme, this corresponds to case 2 . We easily calculate

$$
\begin{aligned}
& a_{2}-a_{1}=2(\mu-2) \quad a_{3}-a_{2}=-(15 / 2)(\mu+1 / 3)(\mu-1) \\
& a_{3}-a_{1}=-(15 / 2)(\mu-1 / 3)(\mu-3 / 5)
\end{aligned}
$$

and

$$
\begin{aligned}
& \alpha_{1}^{+}=15(\mu-1 / 3)(2 / 15-\mu) \\
& \alpha_{1}^{-}=7(\mu-1 / 3) \\
& \alpha_{2}^{+}=15(2 / 3-\mu)(\mu+1 / 3) \\
& \alpha_{2}^{-}=5(\mu+1 / 3)
\end{aligned}
$$

In order to distinguish the different subcases, we have to divide the $\mu$-axis into different subintervals:

$$
\underline{\mu<-1 / 3:} \quad \varepsilon_{1}^{+}=\varepsilon_{1}^{-}=-1, \quad \varepsilon_{2}^{+}=\varepsilon_{2}^{-}=-1 .
$$

Subcase $(\mathrm{d})$ is realized, and because $\left(a_{2}-a_{1}\right)=2(\mu-2)$, we find ind $\boldsymbol{f}_{1}^{ \pm}=-\operatorname{ind} \boldsymbol{f}_{2}^{ \pm}=1$, ind $\left( \pm e_{3}\right)=1$.

$$
-1 / 3<\mu<2 / 15: \quad \varepsilon_{1}^{+}=\varepsilon_{1}^{-}=-1, \quad \varepsilon_{2}^{+}=\varepsilon_{2}^{-}=+1 .
$$

Again subcase $(\mathrm{d})$ is realized with ind $\boldsymbol{f}_{1}^{ \pm}=\operatorname{ind} \boldsymbol{f}_{2}^{ \pm}=1$, ind $\left( \pm \boldsymbol{e}_{3}\right)=-1$.

$2 / 15 \leqq \mu<1 / 3: \quad \varepsilon_{2}^{+}=\varepsilon_{2}^{-}=1, \quad \varepsilon_{1}^{ \pm}= \pm 1, \quad \varepsilon_{3}=1$.

Hence, subcase (c) is realized with ind $\left( \pm e_{3}\right)= \pm 1 . f_{2}^{ \pm}$exists with index 1 .

$1 / 3<\mu<2 / 3$ : Same as previous case, with north and south pole interchanged. $2 / 3 \leqq \mu: \quad \varepsilon_{1}^{ \pm}= \pm 1, \quad \varepsilon_{2}^{ \pm}= \pm 1, \quad \varepsilon_{3}=-1$.

Corresponds to case (a).

Summarizing, we see that $f_{2}^{ \pm}$exists for $\mu<2 / 3$, with index \pm 1 for $-1 / 3<\mu<$ $2 / 3$ and index -1 for $\mu<-1 / 3$. $f_{1}^{ \pm}$exists for $\mu<2 / 15$, always with index 1 . The north pole is elliptic for $\mu<-1 / 3,2 / 15 \leqq \mu<1 / 3$, and $\mu \geqq 2 / 3$, whereas the south pole is elliptic for $|\mu|>1 / 3$. An easy calculation using formula (9) yields

$$
\begin{aligned}
& \boldsymbol{f}_{1}^{ \pm}=(3 / 5-\mu)^{-1}\left( \pm[(28 / 15)(2 / 15-\mu)]^{1 / 2}, 0,1 / 3+\mu\right) \\
& \boldsymbol{f}_{2}^{ \pm}=(1-\mu)^{-1}\left(0, \pm 2[(1 / 3)(2 / 3-\mu)]^{1 / 2},-1 / 3+\mu\right) .
\end{aligned}
$$

The relation between the flow on our unit sphere and the flow induced in the $\xi-\eta$-plane of Ref. [1] (see pictures 3-9 of Ref. [1]) is exactly described by the modification of the stereographic projection introduced earlier in our paper [compare Fig. 1 and formulae (2.25)]. However, we have to keep in mind that this projection is not continuous at the north pole. Indeed, the image of the north pole 
is the circle of radius $(2 L)^{1 / 2}=2 J^{1 / 2}$ in the $\xi-\eta$-plane. Thus, if the north pole is a c.p., as is the case in the Hénon-Heiles model, it gets lost in the process of projection.

Case 3. $\boldsymbol{b}=0$.

In this case the motion on $S^{2}$ is the same as the motion of the endpoint of the angular momentum vector (normalized to unit length) in the case of a top with principal moments of inertia $a_{1}^{-1}, a_{2}^{-1}, a_{3}^{-1}$, as seen from an observer that is fixed with respect to the frame formed by the principal axis of inertia. Notice that if not all $a_{k}$ 's are positive, they can always be made so by a transformation of type (3). Also, by a suitable transformation of type (1.15) we can achieve a permutation of the coordinate axes in such a way that the $a_{k}$ 's are ordered in the same way as their subscripts, i.e.,

$$
a_{1}<a_{2}<a_{3} .
$$

The motion is governed by the Euler equations:

$$
\begin{aligned}
& \dot{x}=J\left(a_{2}-a_{3}\right) y z \\
& \dot{y}=J\left(a_{3}-a_{1}\right) z x \\
& \dot{z}=J\left(a_{1}-a_{2}\right) x y .
\end{aligned}
$$

They possess the six c.p.'s $\boldsymbol{f}_{k}^{ \pm}=\boldsymbol{e}_{k}(k=1,2,3)$, where $\boldsymbol{f}_{1}^{ \pm}, \boldsymbol{f}_{3}^{ \pm}$have an index of one and $f_{2}^{ \pm}$an index of -1 . The solutions of the Euler equations are best labeled with help of the parameter

$$
E=2 K_{2} J^{-2}-a_{0},
$$

and the time $t_{0}$, at which they pass through the $x-z$-plane. The values of $E$ are restricted to the interval $\left(a_{1}, a_{3}\right)$. If we introduce the quantities

$$
\begin{aligned}
m(E) & =\min \left(a_{2}, E\right), \\
M(E) & =\max \left(a_{2}, E\right), \\
p^{2} & =\left(a_{3}-m(E)\right)\left(M(E)-a_{1}\right), \\
k^{2} & =p^{-2}\left(a_{3}-M(E)\right)\left(m(E)-a_{1}\right)<1,
\end{aligned}
$$

the solutions are explicitly given by

$$
\begin{aligned}
& x(t)=\left(\left(a_{3}-E\right) /\left(a_{3}-a_{1}\right)\right)^{1 / 2}\left\{\begin{array}{lll}
\operatorname{cn}\left[p J\left(t-t_{0}\right)\right] & \text { for } & E>a_{2} \\
\operatorname{dn}\left[p J\left(t-t_{0}\right)\right] & \text { for } & E<a_{2},
\end{array}\right. \\
& y(t)=\left(\left(a_{3}-M(E)\right) /\left(a_{3}-a_{2}\right)\right)^{1 / 2}\left(\left(m(E)-a_{1}\right) /\left(a_{2}-a_{1}\right)\right)^{1 / 2} \operatorname{sn}\left[p J\left(t-t_{0}\right)\right], \\
& z(t)=\left(\left(E-a_{1}\right) /\left(a_{3}-a_{1}\right)\right)^{1 / 2}\left\{\begin{array}{lll}
\operatorname{dn}\left[p J\left(t-t_{0}\right)\right] & \text { for } & E>a_{2} \\
\operatorname{cn}\left[p J\left(t-t_{0}\right)\right] & \text { for } & E<a_{2},
\end{array}\right.
\end{aligned}
$$

where $\operatorname{sn} \tau, \operatorname{cn} \tau, \operatorname{dn} \tau$ are the usual Jacobi elliptic functions, i.e., sn $\tau$ is the solution of the initial value problem

$$
d Y / d \tau=\left(1-Y^{2}\right)\left(1-k^{2} Y^{2}\right), \quad Y(0)=0, \quad Y^{\prime}(0)=1,
$$


and $\operatorname{cn}^{2} \tau=1-\operatorname{sn}^{2} \tau, \operatorname{dn}^{2} \tau=1-k^{2} \operatorname{sn}^{2} \tau$. In short, the motion on $S^{2}$ is periodic with frequency

$$
\omega_{1}=(\pi / 2) p J / K(k) \text {, }
$$

where $K(k)$ is the complete elliptic integral of the first kind. Accordingly (compare 2.19 and the remarks following that formula), the motion induced by $K_{2}$ in $R^{4}$ in the case $b=0$ is quasi-periodic with frequencies $\omega_{1}, \omega_{2}$, where $\omega_{1}$ is given in (17) and $\omega_{2}$ differs from the value 1 by a term of order $J$. Again this quasi-periodic motion essentially persists when higher terms are included in the Hamiltonian and the energy is kept sufficiently small. Actually, the proof of a similar theorem in connection with the heavy asymmetric top given by Arnold (Ref. [7]) could be carried over with only minor modifications to the present case.

We note in passing that if $\boldsymbol{b} \neq 0$, the differential equations governing the flow on $S^{2}$ are still the Euler Equations (15), the only difference being, that the dot over $(x, y, z)$ no longer represents the simple derivative with respect to time, but the more complicated operation

$$
d / d t-J I_{\boldsymbol{b}},
$$

where the linear operator $I_{\boldsymbol{b}}$ over $R^{3}$ is defined by

$$
I_{b} \boldsymbol{x}=\boldsymbol{b} \times \boldsymbol{x} .
$$

We therefore obtain the following theorem about the flow that the Hamiltonian $K_{2}$ induces on $S^{2}$ :

Theorem 3. The flow on $S^{2}$ induced by the Hamiltonian $K_{2}$ is the same as the one described by solutions (16) of the Euler equations, but as seen by an observer rotating with angular speed $J|\boldsymbol{b}|$ about an axis with direction $\boldsymbol{b}$ with respect to the standard frame of $R^{3}$.

We turn now to the case in which two of the eigenvalues of $\mathfrak{A}$ coincide. Specifically, we will assume $a \equiv a_{1}=a_{2} \neq a_{3}$.

All other cases of two colliding eigenvalues of the matrix $\mathfrak{A}$ can be reduced to this case, either by a cyclic permutation of the subscripts or by a transformation of type (1.15). By a transformation of type (3), $a$ could be normalized to zero, but not much is gained by such a normalization. Applying the formulas (10), (13) to the present case, we obtain the following simplified formula for the index of a c.p. of the first kind:

$$
\text { ind } e\left(\lambda_{0}\right)=\operatorname{sgn}\left(\lambda_{0}-a_{3}\right) g^{\prime}(\lambda) \text {. }
$$

Case $0^{\prime} . b_{1}^{2}+b_{2}^{2} \neq 0, b_{3}=0$. Let $I$ be the open interval with endpoints $a$ and $a_{3}$ and $I_{0}$ the interior of its complement. $g$ has always two zeros $\lambda_{0}^{ \pm}$in $I_{0}$ and the corresponding c.p.'s are elliptic. Moreover, if the unique relative maximum $G$ of $g$, which occurs at the position

$$
\lambda=\left(b_{3}^{2 / 3} a+\left(b_{1}^{2}+b_{2}^{2}\right)^{1 / 3} a_{3}\right) /\left(b_{3}^{2 / 3}+\left(b_{1}^{2}+b_{2}^{2}\right)^{1 / 3}\right)
$$


in the interval $I$ is positive, another pair of c.p.'s of the first kind is present corresponding to two zeros $\lambda^{ \pm}$of $g$ in $I$. We have

$$
\text { ind } \boldsymbol{e}\left(\lambda^{ \pm}\right)=1 \text {, }
$$

where $\lambda^{-}$is the zero closer to $a$. In any case, of the three possibilities listed in (14) the first or second is realized depending on whether $G<0$ or $G>0$.

Case $1^{\prime} . b_{1}^{2}+b_{2}^{2} \neq 0, b_{3}=0$. The pair of c.p.'s $e\left(a \pm\left(b_{1}^{2}+b_{2}^{2}\right)^{1 / 2}\right)$ is always present. If $g\left(a_{3}\right)<0$, they are both elliptic. If $g\left(a_{3}\right)>0$, the one corresponding to the zero of $g$ that lies closer to $a_{3}$ has an index of -1 , whereas the other one remains elliptic. Moreover, the elliptic pair of c.p.'s $f_{3}^{ \pm}$is present. Of the three possibilities (14) the first or second one is realized, depending on whether $g\left(a_{3}\right)$ is negative or positive.

Case 2'. $b_{1}=b_{2}=0, b_{3} \neq 0$. Besides the elliptic pair of c.p.'s $\boldsymbol{e}\left(a_{3} \pm b_{3}\right)= \pm \boldsymbol{e}_{3}$, which is always present, the circle of fixed latitude $\cos \theta=b_{3} /\left(a-a_{3}\right)$ consists of parabolic c.p.'s (index 0). This circle has a parametric representation

$$
f_{12}(\phi)=\left([g(a)]^{1 / 2} \cos \phi,[g(a)]^{1 / 2} \sin \phi, b_{3} /\left(a-a_{3}\right)\right) \quad(0 \leqq \phi<2 \pi) .
$$

Case $3^{\prime} . \boldsymbol{b}=0$. The same as $2^{\prime}$ except that the pair of c.p.'s $\pm \boldsymbol{e}_{3}$ is of the second kind and $f_{12}(\phi)$ is simply the equator of $S^{2}$ :

$$
\boldsymbol{f}_{12}(\phi)=\cos \phi \boldsymbol{e}_{1}+\sin \phi \boldsymbol{e}_{2} \quad(0 \leqq \phi<2 \pi) .
$$

Remark 1. The case of $3^{\prime}$ occurs in the Hénon-Heiles model for $\mu= \pm 1 / 3$. If $\mu=1 / 3, a_{3}$ and $a_{1}$ collide: we have the two elliptic c.p.'s $f_{2}^{ \pm}= \pm e_{2}$ and the circle of parabolic c.p.'s

$$
f_{31}(\phi)=\cos \phi e_{3}+\sin \phi e_{1} \quad(0 \leqq \phi<2 \pi)
$$

(compare Fig. 7 of Ref. [1]). If $\mu=-1 / 3, a_{3}$ and $a_{2}$ collide. Accordingly, we find the two elliptic c.p.'s

$$
f_{1}^{ \pm}= \pm e_{1}
$$

and the circle of parabolic c.p.'s: $\boldsymbol{f}_{23}(\phi)(0 \leqq \phi<2 \pi)$.

Remark 2. If we normalize $a=0$ by a transformation of type (3), the Hamiltonian in the last two cases becomes

$$
K_{2}=\left(a_{0} / 2\right) J^{2}+b_{3} J M_{3}+\frac{1}{2} a_{3} M_{3}^{2}=J^{2}\left[\left(a_{0} / 2\right)+b_{3} \cos \theta+\frac{1}{2} a_{3} \cos ^{2} \theta\right],
$$

where $a_{3} \neq 0$ (of course $b_{3}=0$ in the case $3^{\prime}$ ).

According to $(2.23) K_{2}$ induces in $R^{4}$ a flow that is quasi-periodic with frequencies

$$
1+(J / 2)\left[a_{0}+b_{3} \cos \theta\right] \text { and } J\left[b_{3}+a_{3} \cos \theta\right],
$$

$\theta$ being an integral of motion. According to Theorem 2 this flow is essentially preserved under perturbations made up of higher order terms, as long as the energy stays sufficiently small. 
Before concluding this section, let us apply the theory of Section 3 to the Hamiltonian (1), i.e., we want to find a condition on the coefficients of that Hamiltonian which guarantees that a certain stable c.p. on $S^{2}$ represents a family of stable periodic solutions of the equations associated with the full Hamiltonian (compare Theorem 1).

As already pointed out in Section 3, it is no loss of generality to assume that $\boldsymbol{e}=-\boldsymbol{e}_{3}$ and $A_{12}=0$. These requirements translate into the following conditions on the coefficients of the Hamiltonian (1)

$$
b_{1}=a_{13}, \quad b_{2}=a_{23}, \quad a_{12}=0,
$$

where the $a_{i k}$ 's are the entries of the matrix $\mathfrak{A}$. (Notice that quite generally, the partial derivative $A_{i k}$ of Section 3 agrees with the corresponding entry of the matrix $\mathfrak{A}$ that we introduced in this section.) Also, from (3.2), we obtain

$$
\lambda=a_{33}-b_{3} .
$$

It is now clear that the decisive expression (3.5), when applied to the Hamiltonian (1), stays exactly as it stands, provided that all $A_{i k}$ 's are replaced by the corresponding $a_{i k}$ 's.

In particular, we have

$$
A=\lambda-a_{11}, \quad B=\lambda-a_{22}, \quad C=-b_{3} .
$$

If we further assume that $\mathfrak{A}$ is diagonal, but not a multiple of the unit matrix, we are again dealing with one of the cases $2,3,2^{\prime}$ or $3^{\prime}$ that we discussed earlier. Using the notation that we introduced in connection with the case 2 , we find

$$
A=\alpha_{1}^{-}, \quad B=\alpha_{2}^{-},
$$

and because $\alpha_{1}^{-} \alpha_{2}^{-}>0$, whenever the south pole is a stable c.p., we may divide the expression (3.5) by $4 \alpha_{1}^{-} \alpha_{2}^{-}$and we obtain

$$
\left(\alpha_{1}^{-} \alpha_{2}^{-}\right)\left(\alpha_{1}^{-}+\alpha_{2}^{-}-b_{3}\right)+(3 / 4) b_{3}\left(\alpha_{1}^{-}+\alpha_{2}^{-}\right)^{2} .
$$

It is no loss of generality if we assume $a_{2}=-a_{1}$, because this can always be achieved by a translation of type (3). The expression (22) is further simplified and becomes

$$
2\left(\alpha_{1}^{-} \alpha_{2}^{-}\right) a_{3}+3 b_{3} a_{1}^{2} .
$$

Inducing a rotation about the vector $e_{1}+e_{2}$ through $\pi$ by a transformation of type (1.15), we find that the analogous expression for the north pole is

$$
2\left(\alpha_{1}^{+} \alpha_{2}^{+}\right) a_{3}-3 b_{3} a_{1}^{2},
$$

where the stability of the north pole implies $\alpha_{1}^{+} \alpha_{2}^{+}>0$.

Whenever one of the expressions (23), (24) is nonzero and the corresponding pole is a stable c.p., then it represents a family of stable periodic solutions of the equations associated with the full Hamiltonian. If both poles are stable c.p.'s this is at least the case for one pole, because under our assumptions the expressions (23) and (24) cannot vanish simultaneously. 
Calculating these expressions in the Hénon-Heiles model and dividing them by $(5 / 2)\left(1 / 9-\mu^{2}\right)$ (recall that for $\mu= \pm 1 / 3$ both poles are not stable), we obtain

$$
219 \mu^{2}-204 \mu+22
$$

for the south pole and

$$
\begin{aligned}
2(3 \mu-2)(15 \mu-2)\left[15 \mu^{2}-12 \mu-1\right]-9(\mu-2)^{2}= & 1350 \mu^{4}-2160 \mu^{3} \\
& +885 \mu^{2}+12 \mu-44
\end{aligned}
$$

for the north pole. One can show that both polynomials have exactly two real roots, but only the larger one (denoted by $\mu_{n}$ and $\mu_{s}$ for the north and south pole respectively) lies in the range of $\mu$-values for which the corresponding pole is a stable c.p. . We find

$$
\mu_{s}=\left(102+(5586)^{1 / 2}\right) / 219 \approx 4 / 5,
$$

and

$\mu_{n}$ lies in the open interval $(13 / 15,14 / 15)$.

Hence, we obtain the result that, whenever in the Hénon-Heiles model the south or north pole of $S^{2}$ is a stable c.p. of the unperturbed Hamiltonian and $\mu \neq \mu_{\text {s }}$ and $\mu \neq \mu_{n}$ respectively, then it represents a family of stable periodic solutions. Actually, the numerical results of Ref. [1] indicate that this is so even when $\mu$ assumes one of the exceptional values.

As a second and last application of Theorem 1, we shall prove the following result:

Theorem 4. To every stable c.p. of the second kind that the function (5) possesses on $S^{2}$ there corresponds a one-parametric family of stable periodic solutions of the equations associated with the full Hamiltonian (1.12) $(n=2)$.

Proof. As pointed out earlier, it is no loss of generality to assume that the c.p. coincides with the south pole. Because we are dealing with a c.p. of the second kind $\lambda$ is an eigenvalue of the matrix $\mathfrak{A}$ and therefore, we have

$$
D=A B C-a_{13}^{2} B-a_{23}^{2} A=0 .
$$

Stability of the c.p. implies $A B>0$, i.e., an inequality that in combination with $D=0$ implies $C \neq 0$ and

$$
\operatorname{sgn} C=\operatorname{sgn} A=\operatorname{sgn} B \text {. }
$$

Taking these facts into account shows that in the decisive expression (3.5) the third term is zero, the second is always nonzero and the first either vanishes or, if it does not, it has the same sign as the second term. Hence, under the assumptions of Theorem 4, the expression (3.5) never vanishes. Our theorem is proved.

Remark. Notice that the result expressed in Theorem 4 is not limited to the case $n=2$, provided we define a c.p. of the second kind more generally to be the endpoint of a unit vector $\boldsymbol{e}$ satisfying (1.6) with a value of $\lambda$ which coincides with an eigenvalue of the Hessian of $K^{(n)}$, evaluated at $\boldsymbol{e}$. 


\section{Final Remarks}

In this final section we want to demonstrate that statement (ii) of the theorem on p. 313 of Ref. [1] cannot be proved with the help of Moser's twist theorem. To show this we let

$$
\lambda_{1}=\beta_{11}=\beta_{12}=\beta_{22}=0, \quad \lambda_{2}=\lambda_{3}=1 / 4
$$

in the notation of (17) on p. 312 of Ref. [1]. Then $\lambda_{1}^{2}+\lambda_{2}^{2}+\lambda_{3}^{2}=1 / 8 \neq 0$ and the Hamiltonian becomes (in our notation)

$$
H=2 J\left(1+M_{2}\right)+\mathcal{O}_{5} .
$$

By a transformation of type (1.5) we induce a rotation about the 1-axis through $\pi / 2$ and obtain

$$
H=2 J\left(1+M_{3}\right)+\mathcal{O}_{5} .
$$

Switching back to the notation of Ref. [1], this Hamiltonian is again of the form (17) (p. 312), but this time the constants are

$$
\lambda_{1}=\lambda_{2}=\lambda_{3}=\beta_{12}=0, \quad-\beta_{11}=\beta_{22}=1 / 2 .
$$

The nondegeneracy condition of Arnold (see p. 313 of Ref. [1]) and Ref. [8] is not satisfied, proving our contention.

Of course, we can also use the methods of Ref. [1] and find that the second derivative in (15) (p. 311) vanishes for the Hamiltonian (1), but the argument given above is much simpler. In order to make the theorem of Ref. [1] a consequence of Moser's twist theorem we suggest to replace the expression $\operatorname{Re}\left(\zeta_{1}^{2} \bar{\zeta}_{1} \bar{\zeta}_{2}\right)$ (in (17) p. 312, Ref. [1]) by $\operatorname{Im}\left(\zeta_{1} \zeta_{2} \bar{\zeta}_{2}^{2}\right)$. In this instance the theorem in question is equivalent (in our notation) to the following statement: If the matrix $\mathfrak{A}$ in the Hamiltonian (4.1) is not a multiple of unity, then the corresponding full Hamiltonian (1.12) possesses infinitely many tori on each energy surface.

The argument for why this theorem seems intuitively obvious runs as follows: The Hamiltonian (4.1) always possesses at least two elliptic c.p.'s on $S^{2}$. The only case in which for both c.p.'s the nondegeneracy condition is violated is the case of a rotation of $S^{2}$ into itself, the c.p.'s being the points of intersection of $S^{2}$ with the axis of rotation. But the Hamiltonian (4.1) induces a rotation of $S^{2}$ if and only if $\mathfrak{A}$ is a multiple of unity.

Acknowledgement. This work was supported by a summer faculty fellowship of the University of Toledo (Summer, 1975).

The author would like to express his sincerest thanks to Mrs. Kay Myers who typed the final manuscript and also to his wife who typed a preliminary draft of it.

Note Added in January 1976. The author is indebted to the referees of the present paper for drawing his attention to several papers (Ref. [9-13]) that deal (among other things) with the question of the existence of periodic orbits of resonant non-linear Hamiltonians near an equilibrium point, i.e. of Hamiltonians that (if analyticity is assumed) in suitable canonical coordinates $(x, y) \in R^{2 n}$ have a convergent power series expansion:

$$
H(x, y)=H_{2}(x, y)+H_{3}(x, y)+\ldots
$$

about the origin of $R^{2 n}$. Here $H_{p}(p=2,3, \ldots)$ is homogeneous in $(x, y)$ of degree $p$ and it is assumed that there exists some rational dependence between the eigenvalues of the infinitesimal transformation 
associated with $\mathrm{H}_{2}$ that is not forced upon them by its symplectic character. In Ref. [9] (p. 634) it is assumed that

$$
H_{2}=\sum_{k=1}^{n}\left(x_{k}^{2}+y_{k}^{2}\right),
$$

and the periodic orbits of $H$ on the energy surfaces of $H$ near the origin of $R^{2 n}$ are associated with the non-degenerate critical points of the function over $P^{n-1}(C)(=$ complex projective space) that is obtained by averaging $H_{4}$ over the flow induced by $H_{2}$ on $S^{2 n-1}$. Because $P^{1}(C)$ is homeomorphic to $S^{2}$ our paper essentially carries through the program outlined in (Ref. [9]) for the case $n=2$ with the difference, however, that the average over $\mathrm{H}_{4}$ is replaced by Birkhoff-Gustavson averaging (which involves also $\mathrm{H}_{3}$ ). The fact that Birkhoff-Gustavson averaging is a valuable tool in the search for periodic orbits near an equilibrium of a Hamiltonian system has been amply demonstrated by Sweet and Schmidt (see e.g. Ref. [10] and references listed there) who simplify and generalize earlier work by Roel (Ref. [11]).

Our approach which makes full use of the group of symplectic transformations leaving $H_{2}$ invariant [in our case $U(2)$ ] can also be applied to other resonant Hamiltonians. E.g. in the case in which the frequencies are opposite equal, $U(2)$ is replaced by $U(1,1)$ (compare Ref. [14] where the same approach is used to interpret the results of a certain computer study).

To discuss the relationship between our work and the very general approach to the question of periodic orbits in Hamiltonian systems initiated by Weinstein (Ref. $[12,13]$ ) is beyond the scope of this note.

\section{References}

1. Braun, M.: On the applicability of the third integral of motion. J. Diff. Eq. 13, 300-318 (1973)

2. Gustavson, F.: On constructing formal integrals of a Hamiltonian system near an equilibrium point. Astron. J. 71, 670-686(1966)

3. Moser, J. K.: Lectures on Hamiltonian systems. Mem. Amer. Math. Soc. 81, 000 000 (1968)

4. Siegel, C.L., Moser, J.K.: Lectures on celestial mechanics. Berlin-Heidelberg-New York: Springer 1971

5. Moser, J.K.: On invariant curves of area preserving mappings of the annulus. Nachr. Akad. Wiss. 1, $1-20(1962)$

6. Hénon, M., Heiles, C.: The applicability of the third integral of motion; some numerical experiments. Astron. J. 69, 73-79 (1964)

7. Arnold,V.I.: Small denominators and problems of stability of motion in classical and celestial mechanics. Usp. Math. Nauk. 18, 91-196 (1963); Russian Math. Surv. 18, 85-193 (1963)

8. Arnold, V.I.: The stability of the equilibrium position of a Hamiltonian system of ordinary differential equations in the general elliptic case. Dokl. Akad. Nauk. USSR 137, 255-257 (1961); Soviet Math. 2, 247 (1961)

9. Moser, J.: Regularization of Kepler's problem and the averaging method on a manifold. Commun. Pure Appl. Math. 23, 609-636 (1970)

10. Schmidt,D., Sweet,D.: A unifying theory in determining periodic families for Hamiltonian systems at resonance. J. Diff. Eq. 14, 597-609 (1973)

11. Roels, J.: An extension to resonant case of Liapunov's theorem concerning periodic solutions near a Hamiltonian equilibrium. J. Diff. Eq. 9, 300-324 (1971)

12. Weinstein, A.: Normal modes for nonlinear Hamiltonian systems. Inventiones math. 20, 47-57 (1973)

13. Weinstein, A.: Lagrangian submanifolds and Hamiltonian systems. Ann. Math. 98. 377-410 (1973)

14. Kummer, M.: An interaction of three resonant modes in a nonlinear lattice. J. Math. Anal. Appl. 52, 64-104 (1975)

\section{Communicated by J. Moser}

Received October 24, 1975 
